\title{
CHLORITE EXAMINATION BY ULTRAMICROTOMY AND \\ HIGH RESOLUTION ELECTRON MICROSCOPY
}

J. I. Brown and M. L. Jackson

For Clays and Clay Minerals

Table of Contents*

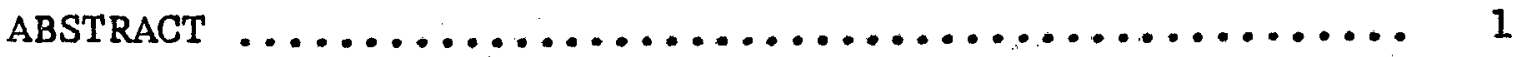

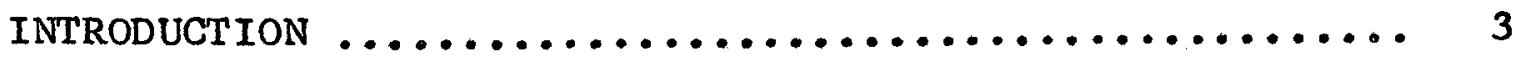

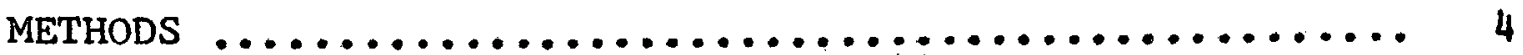

RESULTS AND DISCUSSION $\ldots \ldots \ldots \ldots \ldots \ldots \ldots \ldots \ldots \ldots \ldots$

Intermediate magnification (Fig. la) ........... 6

High magnification (Fig. 1b, c - $13.9 \AA$ spacings) . 6

(Fig. $2 a, b-7.23 \AA$ spacings of equal intensity)

Crystal demage in the electron beam ............ 10

(Fig. 2c, d - 13.9 $\AA$ and some $I_{7.22} \AA<I_{13.9} \AA$ )

Thickness and crystal perfection of the

chlorite particles $\ldots \ldots \ldots \ldots \ldots \ldots \ldots \ldots \ldots \ldots$

CONCLUSIONS $\ldots \ldots \ldots \ldots \ldots \ldots \ldots \ldots \ldots \ldots \ldots \ldots \ldots \ldots \ldots \ldots \ldots$

Acknowledgments $\ldots \ldots \ldots \ldots \ldots \ldots \ldots \ldots \ldots \ldots \ldots \ldots \ldots \ldots$

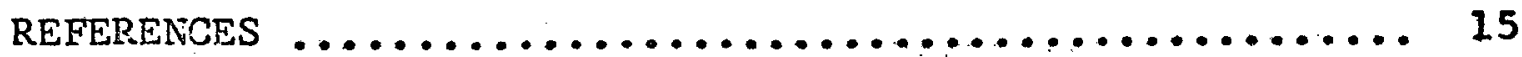

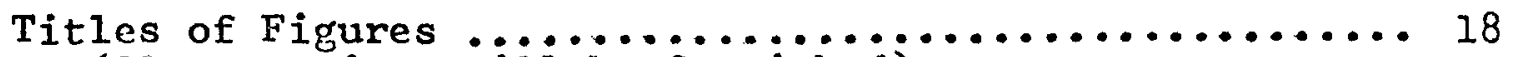

(Glossy prints wili be furnished)

*For editorial purposes, not for publication. Glossy prints will be furnished of figures. 


\section{DISCLAIMER}

This report was prepared as an account of work sponsored by an agency of the United States Government. Neither the United States Government nor any agency Thereof, nor any of their employees, makes any warranty, express or implied, or assumes any legal liability or responsibility for the accuracy, completeness, or usefulness of any information, apparatus, product, or process disclosed, or represents that its use would not infringe privately owned rights. Reference herein to any specific commercial product, process, or service by trade name, trademark, manufacturer, or otherwise does not necessarily constitute or imply its endorsement, recommendation, or favoring by the United States Government or any agency thereof. The views and opinions of authors expressed herein do not necessarily state or reflect those of the United States Government or any agency thereof. 


\section{DISCLAIMER}

Portions of this document may be illegible in electronic image products. Images are produced from the best available original document. 


\section{CHLORITE EXAMINATION BY ULTRAMICROTOMY AND}

HIGH RESOLUTION ELECTRON MICROSCOPY

\section{J. L. Brown}

Analytical Instrumentation Laboratories, Engineering Experiment Station; Georgia Institute of Technology, Atlanta, Georgia 30332 and

M. L. Jackson

Department of Soil Science, University of Wisconsin Madison, Wisconsin 53706 Received 1972

Abstract--Mafic chlorite from Benton, AR was comminuted by high speed rotary blending of a suspension. The particle size fraction less than 2 micrometers in diameter was separated by sedimentation in $\mathrm{H}_{2} \mathrm{O}$. Droplets of suspension of the $<2 \mu \mathrm{m}$ fraction were dried on a layer of Epoxy resin and then additional Epoxy was added and heat-cured to form a resin sandwich. Cross-sections 600 to $900 \AA$ thickness were made of this sandwich on a Reichert automated ultramicrotome. The sections were collected on standard electron microscope specimen screens, reenforced by vacuum evaporated $C$, and examined by transmission electron microscopy (TEM) with a Phillips EM 200 electron microscope with a "microgun" source to minimize heating of the specimen and to improve contrast and high resolution (HREM). Images of the (001) chlorite 
crystallographic planes spaced at $13.9 \AA$ intervals were visible on many of the particle sections. Imaging of the planes depended upon their being nearly parallel to the electron beam (within $0^{\circ} 19^{\prime}$ ) and, therefore, many particles having other orientations did not show the $13.9 \AA$ lattice HREM image. Micrographs made before an appreciable irradiation by the electron beam revealed images of the $7.22 \AA$ structural planes of chlorite, representing the distance between the $(002)$ planes of atomic nuclei in interlayer hydroxyl layers and hydroxyl layers. Loss of the images at $7.22 \AA$ intervals and reenforcement of those at $13.9 \AA$ intervals was caused by the electron beam heating of the chlorite. This is analogous to the well-known partial loss of interlayer hydroxyl on heating at 550 to $760^{\circ} \mathrm{C}$ with 1 oss of the $7.2 \AA$-ray diffraction peak of chlorite and reenforcement of a $13.9 \AA$ spacing. 


\section{INTRODUCTION}

The interlayer surfaces of phyllosilicates are the sites for intercalation of not only hydrated exchangeable cations but also of hydroxy-metal layers such as those of $\mathrm{Fe}, \mathrm{Al}$, and Mg, for which chlorite (Pauling, 1930) is a model mineral. These interlayer configurations are important in cation exchange of radioactive and other ions (Rich, 1968; Syers et al., .1972) and other clay transformations (Jackson, 1963, 1965). Direct photography of ultramicrotomed sections of the crystals examined by high resolution electron microscopy (HREM) have revealed the configuration of the layers and interlayers of the phyllosilicates, chrysotile (Yada, 1967) and muscovite (Brown and Rich, 1968). The pyridine intercalated complex of $\mathrm{TS}_{2}$ has similarly been photographed (Fernandez-Moran et al., 1971).

Margins of montmorillonite particles showed scrolled portions in which the layer intervals were observed edge-on as parallel fringes at $10 \AA$ (Barclay and Thompson, 1969) and 18 to $40 \AA$ with organic complexes (Suito et al., 1969). Imogolite fiber cross sections were observed as thin tubes about $19 \AA$ in diameter in ultramicrotomed sections (Wada et al., 1971). In electron microscope studies of replicas of ferruginous interlayer coatings of micaceous vermiculite (Roth et al., 1967), chlorite was included as a control, for which photographs of crystal layers and hydroxyl interlayers were ultramicrotomed and directly photographed (Brown and Jackson, 1970) by high resolution electron microscopy (UM-HREM) in the present study. 
METHODS

A mafic chlorite from Benton, AR (courtesy of $S$. I. Chapman) was ground in suspension in distilled water with a high speed rotary blender. A suspension of the particle size fraction less than $2 \mu \mathrm{m}$ in equivalent spherical diameter was separated by centrifugation. The sample gave a sharp $14.4 \AA$ basal $x$-ray diffraction peak and higher integral order peaks for chlorite.

Droplets of the chlorite suspension in water were dried on a cured Epoxy (Araldite 6005 from Cargille Sons, 194 Second Ave., Little Falls, NJ) layer to obtain parallel orientation of the clay particles. Additional Epoxy solution was then added and heat cured at $48^{\circ} \mathrm{C}$ overnight (about 12 hours) to form an Epoxy sandwich (Brown and Rich, 1968). A small block was sawed from the Epoxy, suitably shaped by filing, then sectioned by means of a Reichert OmU2 ultramicrotome (Wm. J. Hacker and Co., Inc., P. O. Box 646, West Caldwell, NJ) with a diamond knife. The section was made perpendicularly across the sediment plane so as to cut through the flat faces of the platy particles. The particle platelets and $(001)$ crystal planes in the section thus were approximately normal to the section surface. The thin sections were 600 to $900 \AA$ thickness, as judged from the Sorvall (Norwalk, Conn., 06852) color chart for reflected light. The thin sections were collected from the water-filled 
boat by touching them with the flat surface of a standard electron microscope grid previously coated with $0.1 \%$ bovin albumin (Armour Pharmaceutical Co., Kankakee, II) and dried. The specimen was dried in an air stream and then strengthened with a very thin layer of C (about $100 \AA$ thick) by vacuum evaporation, and examined by transmission electron microscopy (TEM) with a Philips EM200 electron microscope with a "microgun" source to minimize heating of the specimen and to improve contrast and resolution. An accelerating voltage of $100 \mathrm{kv}$ was employed, giving an effective electron beam wavelength of $0.037 \AA$. A $50-\mu \mathrm{m}$ back focal plane objective aperture was employed, with which the angular aperture of the microscope is sufficient to pass Bragg reflections from crystal spacings as close as $4 \AA$. Eastman-P426 $35-\mathrm{mm}$ film was employed for the electron micrographs. 
Intermediate magnification

Insert

The sectioned chlorite platelets distributed in the Fig. 1 Epoxy resin are visible (Fig. $1 a$ ) at intermediate magnification. During sectioning, the Epoxy tears in places (Fig. la) and the clay platelets sometimes fall out. The carbon film helps to prevent this. The $C$ film is essential to ground out the electrostatic charge that tends to build up on the particles in the electron beam. This keeps the Epoxy film mount intact during the examination in the electron microscope. Without the $\mathrm{C}$ layer, the Epoxy film quickly ruptures during the electron microscopic examination. Larger than $2 \mu \mathrm{m}$ phyllosilicate particles tend to be torn from the section by the diamond knife instead of being cut. Particles much smaller than $1 \mathrm{um}$ tend to cake and not accept Epoxy resin between the individual particles and thus do not remain securely anchored in the cured resin so as to be sectioned. High magnification

Calibration of the electron microscope magnification, based on a replica of an optical diffraction grating, shows the fringe-to-fringe spacing of the HREM images to represent a $13.9 \AA$ spacing (Fig. $1 \mathrm{~b}, \mathrm{c}$ ) in many of the outlines of the sectioned particles. This spacing is equal to the (001) $\mathrm{X}$-ray diffraction spacing of chlorite and corresponds to the interval of the 2:1 phyllosilicate crystallographic layers of chlorite. 
When repid photomicrography of the specimen was carried out so as to minimize the exposure of the crystal in the intense electron beam of the electron microscope required for imaging at high magnification, more closely spaced structural

Insert Fig. 2

images were observed at a $7.22 \AA$ interval (Fig. 2a, b). The

7.22 A image spacing corresponds to the (0ก2) X-ray diffraction spacing of the chlorite, at approximately one-half the interval of the (nO1) images (as will be discussed below).

A crystallographic plane of atoms diffracts (scatters) electrons in much the same way that $\mathrm{X}$-rays are diffracted; however, the electrons are diffracted by interactions with the electric fields within the atom in a basic Rutherford scattering process, whereas the $\mathrm{X}$-rays are scattered by the electron clouds surrounding the atomic nuclei. Electron diffraction is much more effective with fewer crystal planes than is X-ray diffraction. For both types of radiation, the Bragg equation, $\mathrm{n} \lambda=2 \mathrm{~d}$ sin $\theta$, applies. Since the electron wavelengths used are much shorter than the $X$-ray wavelengths ordinarily used for crystal diffraction study, the values for $\sin \theta$ are very much smaller. For the $14 \AA$ (001) and $7 \AA$ (002) reflecting planes of chlorite, the $\theta$ angle is on the order of $4^{\prime} 30^{\prime \prime}$ and $8^{\prime} 57^{\prime \prime}$, respectively, i.e. a small fraction of a degree. To form an image of a given crystallographic plane sequence, the incident electron beam must have this Bragg angle with respect to the planes of atomic nuclei in the crystal and the objective aperature must be large 
enough to accept the zero order and at least one diffracted beam. Since electron optical imaging by diffraction from the crystal planes depends on having the Bragg angle with respect to the electron beam, many particles (e.g., Fig. lc, arrow), having other tilt angles, do not show the (001) and/or (002) structural lines within the usual mass contrast TEM image. In accordance with the Abbe theory of microscope resolution for periodic structures, it is necessary to combine optically the zero order and at least the first order diffraction beam (Thomas, 1962, p. 52). Because of spherical aberration in the objective lens of the electron microscope, only those diffracted beams which are sufficiently near the objective axis can be successfully used for imaging; otherwise the conditions in the image plane with respect to phase will be such that contrast will be lost.

These observations illustrate the two basic types of TEM image contrast in the electron microscope, i.e. amplitude (mass thickness) contrast and phase contrast. Amplitude contrast is produced by the back-focal-plane objective aperture and includes both mass-thickness and diffraction contrast. This mechanism applies to distances in the object much larger than interatomic spacings. Phase contrast is produced by recombination of direct and scattered rays at the image plane and is the important mechanism for producing images of crystallographic planes (Fig. 1b, c). For essentially amorphous mineral material (as with most biological 
materials) in which the short range order of individual organizational units is smaller than the resolution of the microscope, the contrast in the image is dependent solely on mass thickness differences recorded in ordinary transmission electron microscopy, for example low-mass Epoxy versus clay particles (Fig. 1a) or particles at non-diffracting tilt angles (Fig. lc, arrow). For longer range crystalline order, as in the chlorite sections examined (Fig. 1b, c; 2a, $b, c, d)$, where the crystal structural spacings are larger than the resolution of the electron microscope, the image contrast arises from phase contrast. Phase contrast images of $100 \AA$ spacings in Yu Yen Stone (antigorite) crystals (Brindley et al., 1958) illustrate the HREM technique for superlattice spacings.

Phase changes $(\mathrm{pc}$ ) cause light and dark zones in the chlorite images ( $p c$, Fig. 2b) and left of center in Fig. 2d. These were also noted in micas (Brown and Rich, 1968, pc in their Fig. 1).

Dark spotty (mottled) areas in the chlorite sections (Fig. 1 and 2) are extinction contours caused by diffraction contrast in which many of the diffracted beams are intercepted by the objective aperture and their contribution to the final image is decreased. These effects also appeared as mottling in micaceous vermiculite (Raman and Jackson, 1964). 
Crystal damage in the electron beam

The fringes depicting the $7.22 \AA$ spacing (Fig. 2b, d) of chlorite were absent in most of the electron micrographs and were detected only. when the photographs were made very rapidly in a given specimen area, by the through-focus technique. This relationship was attributed to crystal damage (dehydroxylation of the hydroxyl interlayers of the chlorite) by the intense electron beam irradiation necessary for imaging at high magnification, rather than to a change in the angle of repose of the crystal section. After a short exposure to heat in the electron beam, the electron diffraction leading to the electron optical image formation revealed only the fringes corresponding to the $2: 1$ layers spaced at $13.9 \AA$ (Fig. 1b,c). In an analogous way, loss of the $7.3 \AA$ fringes from chrysotile and change "to the amorphous state" occurred (Yada, 1967, p. 705) as a result of crystal damage by the electron beam irradiation. Crystal damage, concentrated in one part of the crystal (owing to compositional differences or chance) could contribute to difference in resolution of the $7.22 \AA$ spacing images in different parts of the crystal. The disruption by heating of chlorite mineral in the electron microscope beam is analogous to the effect of furnace heating, which causes the well-known loss of $7.2 \AA \mathrm{X}$-ray diffraction peak of chlorite and reenforcement of a $14.1 \AA \mathrm{X}$-ray diffraction peak at $600^{\circ} \mathrm{C}$ and a $13.9 \AA$ peak at $760^{\circ} \mathrm{C}$ (Brindley, 1961, p. 253; Alexiades and Jackson, 1967). 
The 7.22 A periodicity was resolved electron optically in part of some crystals while only the $13.9 \AA$ periodicity showed in other parts of a given crystal (Fig. 2c, d). Since the Bragg angles involved are approximately the same as the angular aperture of the microscope, the size of the angle between the electron beam and the diffracting plane is critical. Since the diffraction angle for $7.22 \AA$ planes is approximately twice that for $13.9 \AA$ planes, a slightly different orientation of a portion of the chlorite crystal might reveal $13.9 \AA$ spacings without showing the $7.22 \AA$ spacings. The disappearance of the $7.22 \AA$ spacings at a certain point along the crystal (Fig. 2c, d) could be caused by slight warping of the crystal which resulted in two different populations of crystal planes, one oriented at a Bragg angle to give $7.22 \AA$ and the other to give $13.9 \AA$ spacings. The electron optics are more difficult to resolve the stmucture images for the $7.22 \AA$ spacing over the whole sectioned area because the permissible angular deviation from Bragg conditions is more critical (Yada, 1967, p. 707) than those for the $13.9 \AA$ fringes. If this effect contributed to resolution of fewer $7.22 \AA$ spacings images in the electron micrographs than $13.9 \AA$ spacing images, the (001) spacing should have remained at the $14.4 \AA$ spacing of unheated chlorite.

The electron microscope beam scattering, which is produced by the atomic nuclei 1ying within the crystallo- 
graphic planes, depends heavily upon the atomic number of the chemical elements in the plane. Scattering by heavy atoms through dynamical diffraction (Heidenreich, 1964, P. 302), can cause a phase change in the wave front of the electron beam which would create corresponding interference (phase contrast) effects in the image plane. The heavier atoms; particularly $\mathrm{Fe}$ in 6-fold coordination in the mafic chlorite, are particularly effective in scattering the first order diffracted beam of electrons which is then focused with the primary beam to form the electron optical image (Thomas, 1962). A higher $\mathrm{Fe}$ concentration in either layers or interlayers, by dynamical diffraction, could make the $13.9 \AA$ image intensity greater than the $7.22 \AA$ image intensity. The 7.22 $\AA$ images from part of the same crystal which shows only the 13.9 $\AA$ images in other parts (Fig. 2c, d) might, therefore, be attributed in part to appropriate phase shifts resulting from a mosaic of varying elemental composition sequences of octahedral cation sheets in interlayers and layers. Interestingly; the $100 \AA$ fringes of antigorite in one case are interspersed with faint fringes at $50 \AA$ (Brindley et al., 1958, their Fig. 1, b). 
Thickness and crystal perfection of the chlorite particles

A large proportion of the chlorite particles $(<2 \mathrm{~mm}$ fraction) are on the order of 150 to $200 \AA$ thick (Fig. 1 and 2). Since the X-ray diffraction peaks at 14.4 and $7.2 \AA$ for this sample were very intense (data not shown herein), the images (Fig. 1 and 2) show that as few as 10 to 20 (001) crystallographic units are ample for development of sharp X-ray diffraction maxima.

No crystal distortion is observable on the outside boundary of the chlorite crystal images (Fig. 1 and 2), indicating that no short range structural damage at the edges was created by surface tension effects or by the mechanical comminution process. No mass contrast image extended beyond the diffraction contrast image. The question as to whether outermost "disturbed" layers and/or edges would be created by comminution in a high speed rotary blender is thus settled negatively for this chlorite. 


\section{CONCLUSIONS}

1. High resolution electron microscopy of ultramicrotomed sections made normal to the basal planes of the chlorite, revealed phase contrast electron optical image fringes of the $7.22 \AA(002)$ atomic plane spacings of chlorite before heating and $13.9 \AA$ spacings after heating.

2. Many more crystal specimens were seen with $13.9 \AA$ spacings than with 7.22 A spacings. Most crystals showed intensities of $13.9 \AA$ fringes which were greater than intensities of the $7.22 \AA$ fringes. A few crystals showed long sequences of equal-intensity fringes at $7.22 \AA$ when the electron micrographs were made rapidly with the throughfocus technique. The $7.22 \AA$ phase contrast image was subject to loss during a few minutes of exposure in the intense electron beam of the microscope operated at 100 $\mathrm{kv}$, leaving reenforced images, corresponding to planes spaced at $13.9 \AA$ as for chlorite furnace heated at $760^{\circ} \mathrm{C}$.

3. Single particles, $<2$ um in equivalent spherical diameter and plate-shaped, were frequently of 10 to 20 unit cells (each of $14 \AA$ ) thickness. This size fraction gave very sharp $\mathrm{X}$-ray diffraction peaks in separate studies.

4. No evidence of a "disturbed" layer, or coatings of short range order only, occurred in the outermost layer or at the ends of the chlorite particles, which had been comminuted by high-speed rotary blending of a suspension. 
Acknowledgments--This cooperative research was supported in part by the College of Agricultural and Life Sciences, University of Wisconsin, under project 1336 , in part by the Analytical Instrumentation Laboratories, Engineering Experiment Station, Georgia Institute of Technology, and in part by the Division of Biology and Medicine, Environmental Sciences Branch, United States Atomic Energy Commission Contract AT (11-1)-1515-Jackson (paper C00-1515-25) and the National Science Foundation grant GP1108-Jackson. Gratitude is expressed to Mr. S. Y. Lee for assistance with the printing and mounting of the electron micrographs.

\section{REFERENCES}

Alexiades, C. A., and Jackson, M. L. (1967) Chlorite determination in clays of soils and mineral deposits: Amer. Mineral. 52, 1855-1873.

Barclay, L. M., and Thompson, D. W. (1969) Electron microscopy of sodium montmorillonite: Nature 222, 263. Brindley, G. W. (1961) Chlorite minerals: Ch. 6, pp. 242-296, in "X-ray Identification and Crystal Structures of Clay Minerals" G. Brown, ed. The Mineral Society (London).

Brindley, G. W., Comer, J. J., Uyeda, R., and Zussman, J. (1958) Electron-optical observations with crystals of antigorite: Acta Cryst. 11, 99-102. 
Brown, J. L., and Jackson, M. L. (1970) Chlorite examination by ultramicrotomy and high resolution electron microscopy: Abstracts, 19th Clay Miner. Conf., FL, p. 13.

Brown, J. I., and Rich, C. I. (1968) High resolution electron microscopy of muscovite: Science 161, 11351137.

Fernández-Morán, J., Ohstuki, M., Hibino, A., Hough, C. (1971) Electron microscopy and diffraction of layered, superconducting intercalation complexes: Science 174, 498-500.

Heidenreich, R. (1964) Fundamentals of Transmission Electron Microscopy: Interscience, New York, NY. Jackson, M. L. (1963) Interlayering of expansible layer silicates in soils by chemical weathering: Clays and Glay Minerals 11, 29-46.

Jackson, M. L. (1965) Clay transformations in soil genesis. during the Quaternary: Soil Sci. 99, 15-22.

Pauling, L. (1930) Structure of chlorites: Proc. Nat. Acad. Sci., Wash. 10, 692-694.

Raman, K. V. V., and Jackson, M. L. (1964) Vermiculite surface morphology: Clays and Clay Minerals 12, 423429. .

Rich, C. I. (1968) Hydroxy interlayers in expansible layer silicates: Clays and Clay Minerals 16, 15-30. 
Roth, C. B., Jackson, M. L., de Villiers, J. M., and Volk, v. v. (1567) Surface colloids on micaceous vermiculite: Soil Chemistry and Soil Fertility, (Edited by G. V. Jacks). Trans. Comm. II and IV, Int1. Soc. Soil Sci., Aberdeen. Int1. Soc. Soil Sci. publ., pp. 217-221. Suito, E., Arakawa, M., and Yoshida, T. (1969) Electron microscopic observation of the layer of organo-montmorillonite: Proc. 3rd Intl. Clay Conf. (Tokyo) 1, 757-763.

Syers, J. K., Mokma, D. L., Jackson, M. L., Dolcater, D. L., and Rex, R. W. (1972) Mineralogical composition and cesium-137 retention properties of continental aerosolic dusts: Soil Sci. 113,116-123.

Thomas, G. (1962) Transmission Electron Microscopy of Metals: John Wiley, NY. Wada, K., Yoshinaga, N., Yotsumoto, H., Ibe, K., and Aida, S. (1971) High resolution electron micrographs of imogolite: Clay Minerals 8, 847-849.

Yada, K. (1967) Study of chrysotile asbestos by a high resolution electron microscope: Acta Cryst. 23, 704707. 


\section{List of' Figures}

Fig. 1. Transmission electron micrograph of chlorite $(\ll 2 \mu \mathrm{m})$ set in Epoxy plastic and thin-sectioned by a diamond-knife ultramicrotome (a) at intermediate magnification, showing some torn areas in the Epoxy; (b) at high magnification, showing $13.9 \AA$ crystallographic spacings by phase contrast imaging; (c) same, with an area (arrow) not in proper position to show a phase contrast image.

Fig. 2. Transmission electron micrograph of chlorite sections taken rapidly with through-focus technique to minimize specimen heatings (a) showing $7.22 \AA$ spacing; (b) same, with phase change fringes (PC); (c and d) same with areas of 7.22 and $13.9 \AA$ spacings in different parts of the same crystal. 

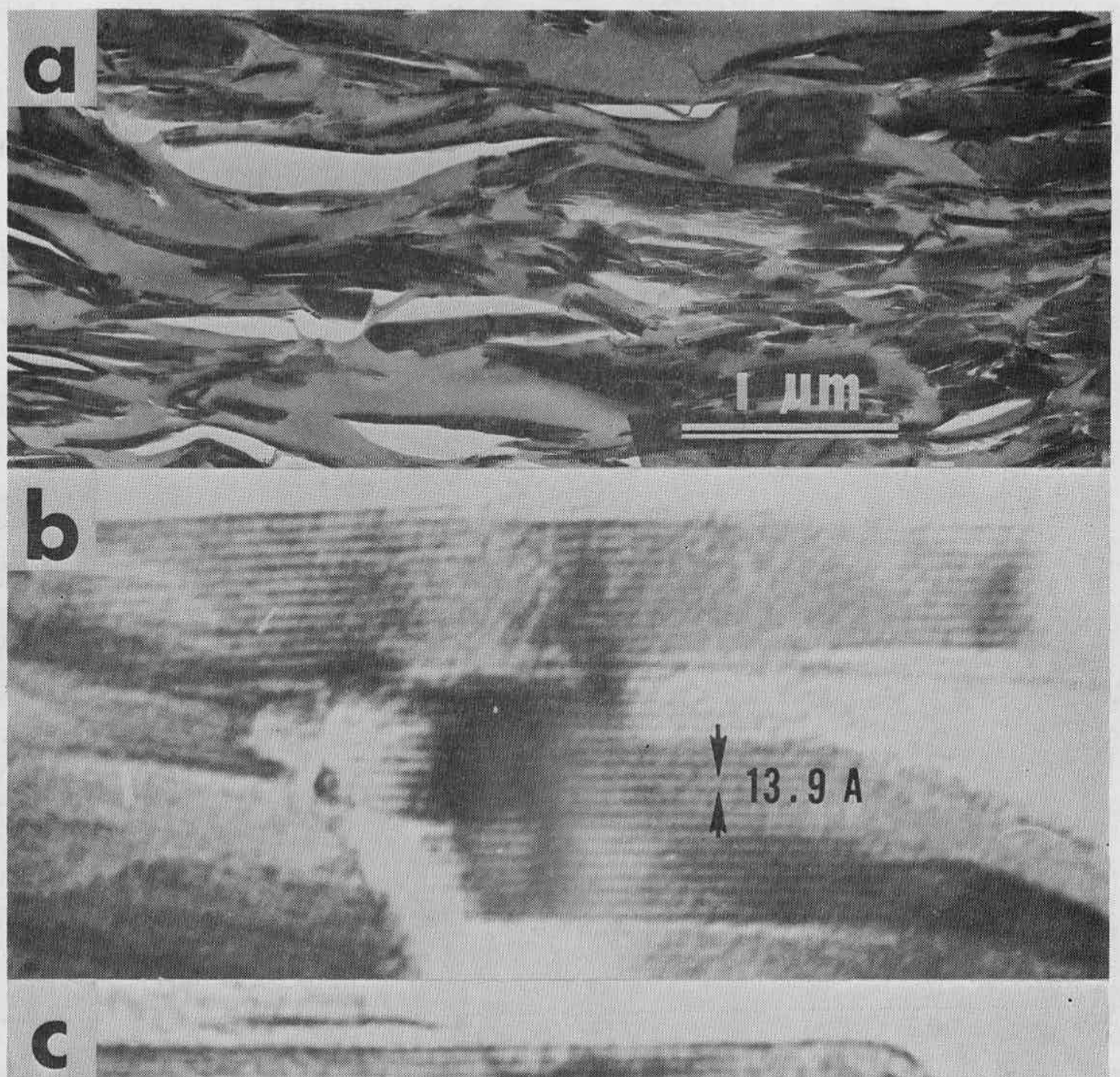

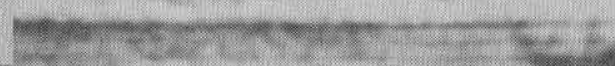

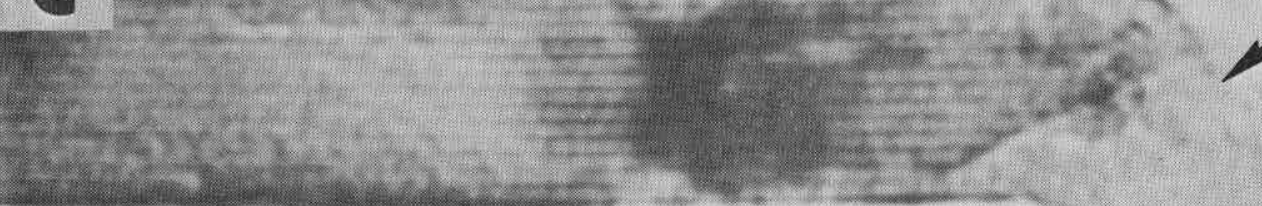

-

$x$

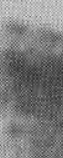

Fig. 1. Transmission electron micrograph of chlorite $(<2 \mu m)$ set in Epoxy plastic and thin-sectioned by a diamond-knife ultramicrotome (a) at intermediate magnification, showing some torn areas in the Epoxy; (b) at high magnification, showing 13.9 \& crystallographic spacings by phase contrast imaging; (c) same, with an area (arrow) not in proper position to show a phase contrast image. 


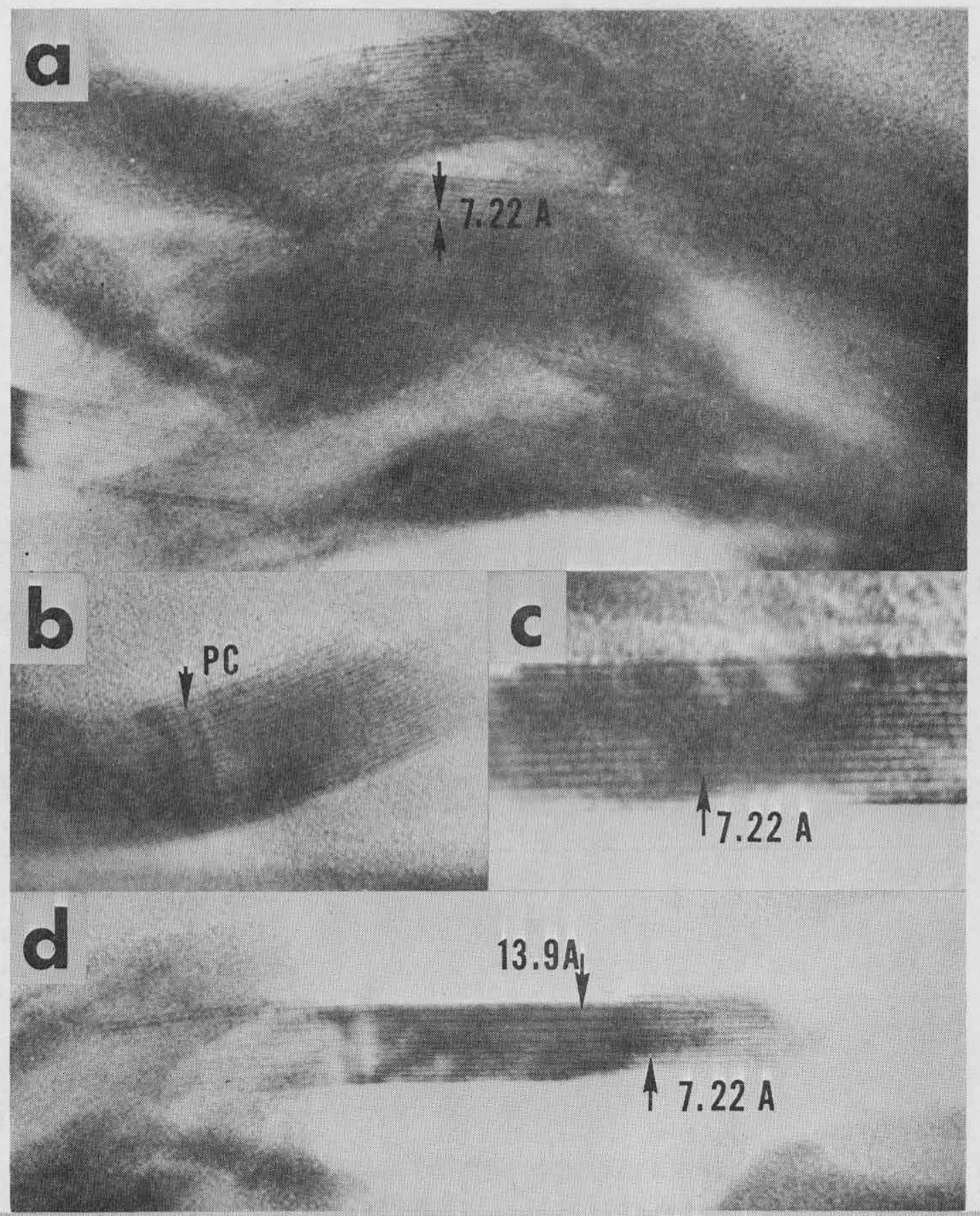

Fig. 2. Transmission electron micrograph of chlorite sections taken rapidly with through-focus technique to minimize specimen heatings (a) showing 7.22 \& spacing; (b) same, with phase change fringes (PC); (c and d) same with areas of 7.22 and 13.9 \& spacings in different parts of the same crystal. 